\title{
X-ray Spectroscopy of Thermally Emitting Neutron Stars
}

\author{
M. H. van Kerkwijk \\ Department of Astronomy \& Astrophysics, University of Toronto, \\ 60 Saint George Street, Toronto, Ontario, M5S 3H8, Canada
}

\begin{abstract}
I describe recent high-resolution X-ray spectroscopy of surface emission from nearby, thermally emitting neutron stars. I focus on RX J0720.4-3125, RX J1308.6+2127 and RX J1605.3+3249, all of which have similar temperature, but differ in the presence and strength of absorption features in their spectra. I discuss possible causes for the absorption we see in two sources, and conclude that it may be proton cyclotron line absorption, but weakened due to the strong-field quantum electrodynamics effect of vacuum resonance mode conversion.
\end{abstract}

\section{Introduction}

ROSAT discovered a number of nearby neutron stars whose emission appears to be entirely thermal, uncontaminated by accretion or magnetospheric processes. At present, six (possibly seven) sources are known (Treves et al. 2000; Haberl 2004). For four sources, optical counterparts have been identified. The high $\mathrm{X}$-ray to optical flux ratios leave no model but an isolated neutron star.

As a class, the sources are interesting because of the implied existence of a fair number of neutron stars different from the usual radio pulsars and Xray binaries. At present, their nature remains unclear. The original idea, of old neutron stars accreting slowly from the interstellar medium, has become unlikely because of the very low accretion rates implied by high proper motions. Instead, they might be radio pulsars beamed away from us, although in this case the long periods are surprising. Perhaps they have very strong magnetic fields, and are descendants of anomalous X-ray pulsars and soft gamma-ray repeaters.

As individuals, the sources are of particular interest because of the opportunity to study uncontaminated emission from a neutron-star atmosphere. The hope is that this will allow one to infer precise values of the temperature, surface gravity, gravitational redshift and magnetic field strength. In turn, these could be used to constrain the interior, and hence learn about the equation of state of cold, ultradense matter, an unexplored region in QCD parameter space.

\section{X-ray Spectra}

Given the interest, long spectroscopic observations were taken with the Chandra $X$-ray Observatory and XMM-Newton. The first results were discouraging: no lines were found in XMM spectra of RX J0720.4-3125 (Paerels et al. 2001), nor 


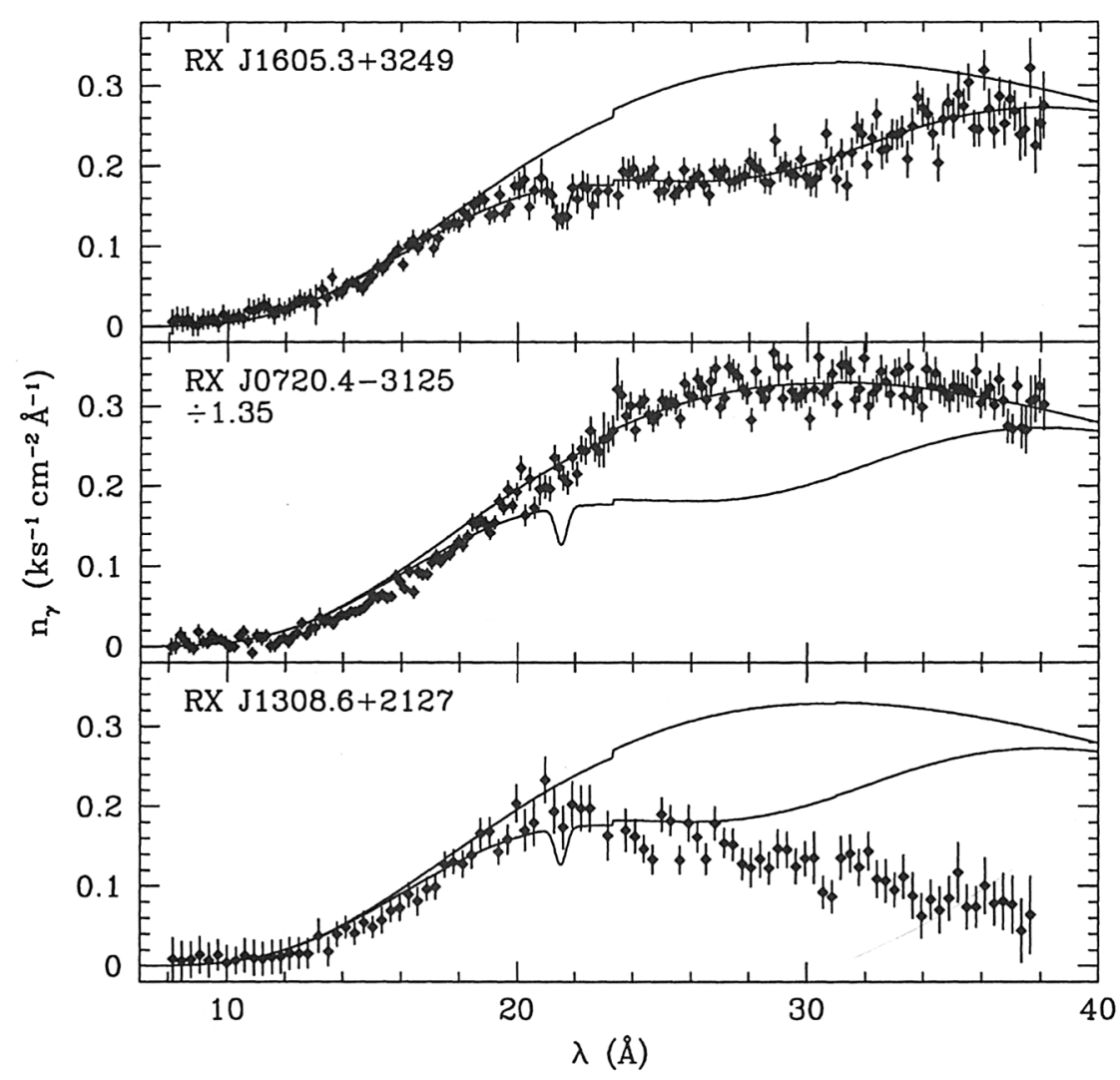

Figure 1. RGS spectra of three nearby, thermally emitting neutron stars. The top panel shows RX J1605.3+3249, for which the spectrum shows clear evidence for an absorption feature. Overdrawn is the best-fit model, a slightly extincted black body with two Gaussian absorption features. The second and third panel show the spectra of RX J0720.4-3125 and J1308.6+2127, with the same model overdrawn for comparison. Both have similar temperatures, but RX J0720.4-3125 has no absorption, while RX J1308.6+2127 has much stronger absorption.

in Chandra spectra of the prototype of the class, RX J1856.5-3754 (Burwitz et al. 2001), not even after $500 \mathrm{ks}$ (Drake et al. 2002; Braje \& Romani 2002). Instead, for both sources the spectra were found to be remarkably well described by mildly extincted black bodies. This was unexpected, since for light-element atmospheres one would expect a hard tail (because the opacities decrease towards higher energies), while for anything else one would expect to see lines (for reviews, see Pavlov, Zavlin, \& Sanwal 2002; Zavlin \& Pavlov 2002).

The situation changed this year. For RX J1308.6+2127, the fourth-brightest object in the class, Haberl et al. (2003) discovered a broad absorption feature in $X M M$ data. It extends from $\sim 0.5 \mathrm{keV}$ to lower energies, and can be described by a Gaussian centered at $\lesssim 0.3 \mathrm{keV}$. Furthermore, the third-brightest object, 
RX J1605.3+3249, was found to show a weaker, less wide absorption feature centered at a somewhat higher energy of $0.45 \mathrm{keV}$ (van Kerkwijk et al. 2004), as well as a narrower, marginally significant absorption line at $0.55 \mathrm{keV}$.

In Figure 1, the RGS spectra obtained with $X M M$ for both sources are shown. In addition, the spectrum of RX J0720.4-3125 is shown. This source has a very similar temperature, $k T \simeq 90 \mathrm{eV}$, but a featureless spectrum.

\section{Proton Cyclotron Absorption and Vacuum Resonance}

For RX J1308.3+2127, Haberl et al. (2003) suggested that the feature was due to proton cyclotron absorption. Given the energy of $\lesssim 0.3 \mathrm{keV}$, the implied magnetic field strength is $\lesssim 5(1+z) \times 10^{13} \mathrm{G}$ (where $1+z=\left[1-2 G M / R c^{2}\right]^{-1 / 2} \simeq$ 1.3 is the gravitational redshift factor). This is not unreasonable, given the observed slow spin period, $P=10.3 \mathrm{~s}$. From the temperature, the neutron star should be about half a million years old. Equating this to the characteristic age, one infers a current spin-down rate $\dot{P} \sim P / 2 t \sim 3 \times 10^{-13}$, and a magnetic field of $3.2 \times 10^{19}(P \dot{P})^{1 / 2} \sim 6 \times 10^{13} \mathrm{G}$, consistent with what is required.

The large width of the feature, $\sigma_{E} / E \gtrsim 1 / 3$, is as expected for proton cyclotron absorption, since the cyclotron energy scales linearly with the magnetic field strength, which will vary over the surface (by a factor two for a centered dipole). Furthermore, as mentioned by Haberl et al. (2003), the equivalent width is consistent with model calculations of Zane et al. (2001).

If we assume the absorption in RX J1605.3+3249 is due to proton cyclotron absorption as well, we infer $B \simeq 7(1+z) \times 10^{13} \mathrm{G}$. Unfortunately, no pulsations have been found - to a limit of $3 \%$ in the frequency range $0.001-800 \mathrm{~Hz}$ (van Kerkwijk et al. 2004) - so we cannot verify this. Puzzling in this case, however, is the difference in strength and width of the features in the two sources. As mentioned, the temperatures are very similar, so that cannot be the explanation.

The fields strengths inferred above are in excess of the critical quantum electrodynamics field $B_{\mathrm{QED}}=4.4 \times 10^{13} \mathrm{G}$, at which the electron cyclotron energy equals the electron rest mass. One effect that may become important is that photons propagating down the density gradient in the atmosphere can change polarization mode at "vacuum resonance", where the plasma contribution to the dielectric properties is compensated by the QED effect of vacuum polarization.

Vacuum resonance has recently been studied in detail (e.g., Lai \& Ho 2003), and it was found to lead to a reduction in the contrast of spectral features when it occurs between the deeper photosphere for the extraordinary mode photons and the shallower one for the ordinary mode photons. For the relevant energies of $\sim 0.6 \mathrm{keV}$ at the surface, this will be the case for magnetic fields in the range $(0.7-50) \times 10^{14} \mathrm{G}$.

If this is correct, it will not be very important for RX J1308.6+2127, and it makes sense that that source's feature could be reproduced by the models of Zane et al. (2001), which do not take vacuum polarization into account. It should affect RX J1605.3+3249, however. And indeed, it may explain why the observed feature is so narrow: we might be seeing only absorption from regions with relatively low field, $B \lesssim 9 \times 10^{13} \mathrm{G}$, the contrast of the absorption in regions with higher field being reduced due to the vacuum resonance. 


\section{Future Prospects}

The discovery of absorption features should help further theoretical work on the vacuum resonance (in progress; Ho \& Lai 2004). In particular, in both sources, the absorption extends up to $\sim 0.5 \mathrm{keV}$. Might it be that the maximum energy out to which absorption is seen is set by vacuum resonance? If so, the observations provide a direct measurement of the critical magnetic field strength: about $7(1+z) \times 10^{13} \mathrm{G}$.

Theoretical work is also needed on neutral hydrogen, which is strongly bound in high magnetic fields (for a review, see Lai 2001). Hence, it should be present, and could lead to observable features. So far, it has only been studied at lower fields (e.g., Pavlov \& Meszaros 1993; Zavlin \& Pavlin 2002). It might possibly also help understand the puzzling overall spectral energy distributions.

Observationally, the obvious steps are a survey of the remaining sources, and using Chandra/LETGS to obtain spectra over a larger wavelength range and with phase resolution. Timing and astrometric studies could help constrain magnetic field strengths, distances, space velocities, places of origin, and ages.

All in all, the future looks bright, even if we may learn more about QED than about QCD.

Acknowledgments. I thank Dong Lai and George Pavlov for the good discussions during the conference, during which the ideas described here arose.

\section{References}

Burwitz, V., Zavlin, V., Neuhäuser, R., Predehl, P., Trümper, J., \& Brinkman, A. C. 2001, A\&A, 379, L35

Braje, T. M., \& Romani, R. W. 2002, ApJ, 580, 1043

Drake, J. J. et al. 2002, ApJ, 572, 996

Haberl, F. 2004, Adv. Sp. Res., 33, 638

Haberl, F., Schwope, A. D., Hambaryan, V., Hasinger, G., \& Motch, C. 2003, A\&A, 403, L19

Ho, W. C. G., \& Lai, D. 2004, ApJ, in press (astro-ph/0402088)

Lai, D. 2001, Rev. Mod. Phys., 73, 629

Lai, D., \& Ho, W. C. G. 2003, ApJ, 588, 962

Paerels, F. et al. 2001, A\&A, 365, L298

Pavlov, G. G., \& Meszaros, P. 1993, ApJ, 416, 752

Pavlov, G. G., Zavlin, V. E., \& Sanwal, D. 2002, in Neutron Stars, Pulsars, and Supernova Remnants, eds. W. Becker et al., (Garching: MPE), p. 273

Treves, A., Turolla, R., Zane, S., \& Colpi, M. 2000, PASP, 112, 297

van Kerkwijk, M. H., Kaplan, D. L., Durant, M., Kulkarni, S. R., Paerels, F. 2004, ApJ, in press (astro-ph/0402418)

Zane, S., Turolla, R., Stella, L., \& Treves, A. 2001, ApJ, 560, 384

Zavlin, V. E., \& Pavlov, G. G. 2002, in Neutron Stars, Pulsars, and Supernova Remnants, eds. W. Becker et al., (Garching: MPE), p. 263 